\title{
Publication Bias and Evidential Value in Speech, Language, and Hearing Research
}

Jean Alexander ${ }^{1}$ James A. Green ${ }^{1,2}$

\author{
${ }^{1}$ School of Allied Health, University of Limerick, Ireland \\ ${ }^{2}$ Health Research Institute, University of Limerick, Ireland
}

Purpose: This research examined the evidential value of research in Speech, Language, and Hearing (SLH), and the extent to which there is publication bias in reported findings. We also looked at the prevalence of good research practices, including those that work to minimize publication bias.

Method: We extracted statistical results from 51 articles reported in four meta-analyses. These were there analyzed with two recent tests for evidential value and publication bias - the $p$-curve and the $Z$-curve. These articles were also coded for pre-registration, data access statements, and whether they were replication studies.

Results: P-curves were right-skewed indicating evidential value, ruling out selective reporting as the sole reason for the significant findings. The $Z$-curve similarly found evidential value but detected a relative absence of null results, suggesting there is some publication bias. No studies were pre-registered, no studies had a data access statement, and no studies were full replication studies (3 studies were partial replications).

Conclusions: Findings indicate SLH research has evidential value. This means that decision-makers and clinicians can continue to rely on the SLH research evidence base to influence service and clinical decisions. However, the presence of publication bias means that meta-analytic estimates of effectiveness may be exaggerated. Thus, we encourage SLH researchers to engage in study pre-registration, make result data accessible, conduct replication studies, and document null findings. 


\section{INTRODUCTION}

\section{Publication Bias and Selective Reporting}

Publication bias is the term used to describe the tendency of journals to favor publishing studies with statistically significant results, at the expense of statistically non-significant results (Sterling, 1959). If publication bias is present in a field, it means that the published literature presents a biased selection of all the studies that have been carried out. Studies with non-significant results are placed in the 'file drawer'; and the publication of only significant results means that published results are biased towards false discoveries (Ioannidis, 2005; Sterling, 1959). In health fields, this biased publication of significant findings leads to overestimation of treatment effects and is a recognized threat to the value of meta-analysis when making policy and treatment decisions (Dwan et al., 2013).

Publication bias and other reporting biases are issues in many research fields, including neuroimaging, animal studies, psychology, and genetics (Ioannidis et al., 2014). Fanelli (2012) analyzed 4,600 papers across all disciplines between 1990 and 2007 and found reported significant findings had increased $22 \%$ over that time. The bias for significant findings was most prevalent in social sciences (e.g., psychology, economics) and in applied disciplines.

Publication bias has led to other 'questionable research practices' (QRPs) to increase the chances of finding a significant — and therefore publishable — result (John et al., 2012). Simonsohn et al. (2014) coined the term ' $p$-hacking' to describe researchers' decisions and behaviors which manipulate data and analyses to produce a significant $p$ value. For example, flexibility in the decision over when to stop collecting data or which outliers to exclude based on how they would affect the $p$-value. Selective reporting refers to researchers' decisions to report results depending on the nature and direction 
of results (Higgins et al., 2021). For example, when a researcher conducts multiple analyses but only reports some of them, depending on the nature and direction of the results (Dwan et al., 2013). The likely outcome of these behaviors are incorrect findings of statistical significance and inflated effect sizes, further contaminating the research literature. These behaviors do not lead just to a loss of published non-significant data, but it also means that published results can be false discoveries (false-positives). Simmons et al. (2011) conducted a study which demonstrated how easy it is to find a false-positive when using QRPs. They found that when there was flexibility in choosing dependent variables, choosing sample size, using covariates, and reporting subsets of experimental conditions, there was a $61 \%$ false-positive rate. Thus, if a researcher employs four QRPs they are more likely than not to have a false-positive result. John et al. (2012) compared QRPs to steroids, saying they artificially enhance findings.

Considering the negative influence of questionable research practices on the validity and integrity of scientific research, why are researchers tempted to partake in them? For many of the practices, they were commonplace, and their negative effects were not well known until recently (Bishop, 2019). However, within the 'publish or perish' culture in academia, where career progression depends in part on the number of papers published (De Rond \& Miller, 2005; Ioannidis et al., 2014), the temptation to engage in QRPs to find a significant result becomes more understandable. John et al. (2012) surveyed 2000 psychologists about these practices and found the majority of respondents engaged in selective reporting of studies, not reporting all dependent measures, collecting more data after determining whether the results were significant, reporting unexpected findings as having been predicted, and excluding data post-hoc. 


\section{Tests for Publication Bias}

There are a range of publication bias tests available but no 'gold standard'. Some common tests for meta-analyses include funnel plots (Egger et al., 1997; Light \& Pillemer, 1984), fail-safe N (Rosenthal, 1979), trim and fill analysis (Duval \& Tweedie, 2000), Egger's regression test (Egger et al., 1997), Begg's test (Begg \& Mazumdar, 1994) and Macaskill's test (Macaskill et al., 2001). Visual tests like funnel plots have been favored for their simplicity but have also faced criticism for being subjective and that different observers may interpret them differently (Greenland, 1994).

The tests of publication bias described so far were all designed for meta-analyses. Recently, however, several new tests have been developed that test for publication bias for broader and more heterogenous bodies of research literature. The $p$-curve is claimed to detect 'evidential value' - that the set of results are robust to publication bias - with $20 p$-values even when the set of studies is only powered at $50 \%$ (Simonsohn et al., 2014). Further, with 'just five $p$-values, $p$-curve has more power than the individual studies on which it is based' (p. 544). The $p$-curve achieves this by plotting reported $p$-values for the focal hypotheses of a study. The $p$-values should show a strong right skew — that is, if there is evidential value in a set of findings, smaller $p$ values $(p<.01)$ should be more likely than $p$-values closer to $p=.05$ (See top-left panel of Figure 1). In contrast, if the distribution of $p$-values is flat, lumpy, or bunched around $p=.04-.05$, then the set of studies has no evidential value (top-right panel of Figure 1).

However, the $p$-curve has been criticized as less robust to studies where multiple dependent measures were collected, but only significant measures were reported (Bishop \& Thompson, 2016). Further, as the $p$-curve does not include non-significant results, it does not provide information directly on selection bias, and may overestimate power in the studies analyzed (Brunner \& Schimmack, 2020). 
An new alternative measure of estimating publication bias and replicability is the $Z$ curve (Bartoš \& Schimmack, 2020b; Brunner \& Schimmack, 2020). In contrast, to the $p$ curve, it also considers non-significant results. If there is no selection bias, then $Z$-scores derived from published $p$-values should produce the familiar bell curve (bottom-left panel of Figure 1). However, where there is selection for significant results, the curve is truncated at the significance criteria $(\mathrm{p}<.05$ or $\mathrm{Z}=1.96$; bottom-right panel of Figure 1).

Evidential value;

less publication bias
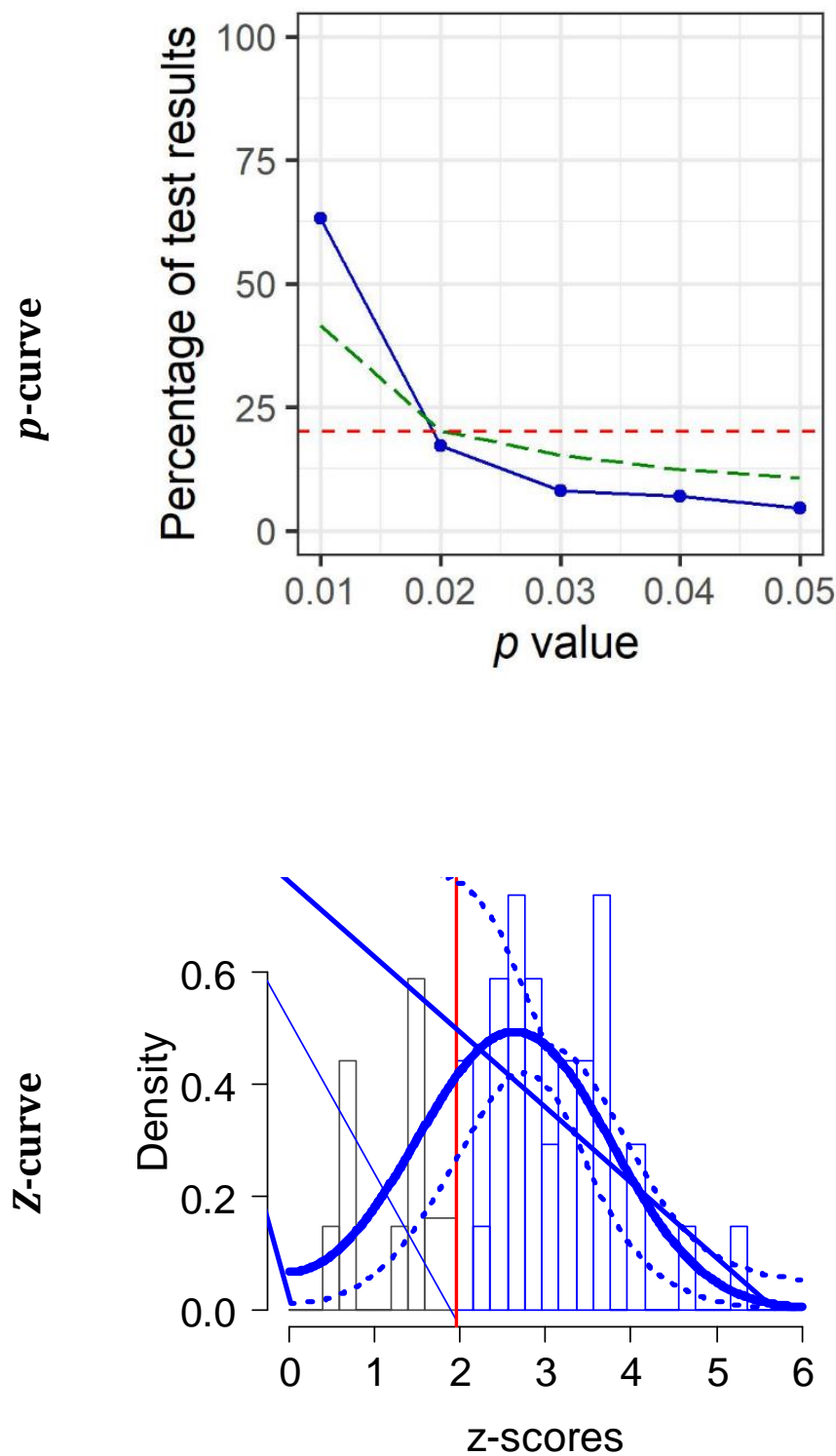

Lower evidential value;

more publication bias
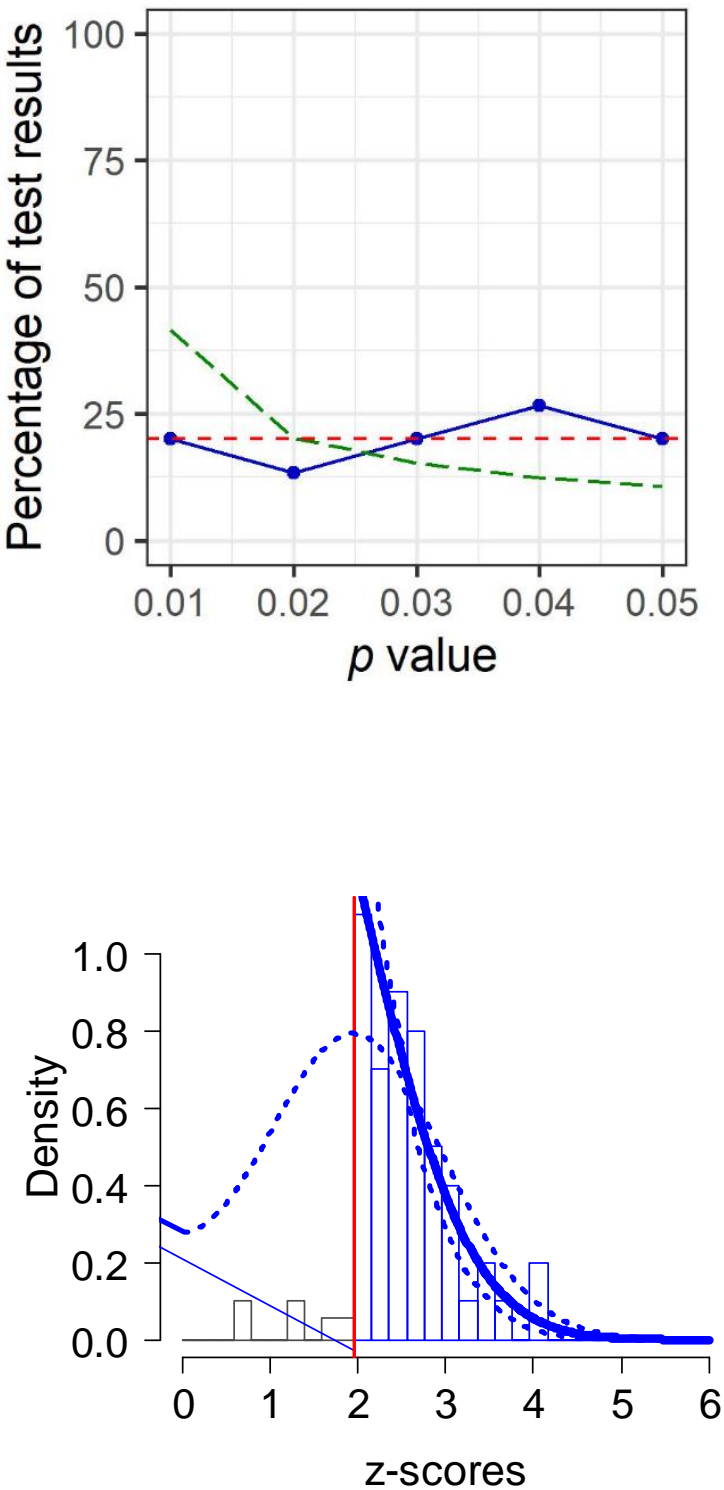
Figure 1: The top panels are demonstration p-curves. The observed distribution of p-values is plotted in blue, a null distribution in red, and 33\% power in green. In the top-left panel, the observed blue shows a clear right skew, indicating evidential value. In contrast, in the top-right panel, the blue observed line is flat/lumpy, and shows no skew, hence no evidential value. For the Z-curve (lower panels), where there is good evidential value and little publication bias (lower-left), the tests should approximate the bell curve, and the distribution should continue to the left of the red line $(Z=1.96, p=.05)$. In contrast, on the lower-right, there are almost no studies to the left of the red line, and no bell curve is formed, suggesting poor evidential value and strong evidence of publication bias.

\section{Publication Bias in Speech, Language and Hearing Research}

As discussed above, publication bias and questionable research practices, including selective reporting, have been found to be issues across many disciplines. However, speech, language and hearing (SLH) research has not yet been examined for selective reporting. Chow (2018) examined whether SLH researchers search for and include unpublished literature and test for publication bias when conducting meta-analyses. He found that less than $50 \%$ of meta-analyses included unpublished literature or tested for publication bias, and only 38\% interpreted the findings of the publication bias tests. However, he did not test a set of SLH studies for selective reporting. SLH research evidence is used to inform policy decisions, resource allocation and what treatments to use in SLH (American Speech-Language-Hearing Association, 2005) and therefore, it is important that these studies reflect genuine results. Therefore, this meta-science review aims to:

1. determine whether research in American Speech-Language-Hearing Association journals has good evidential value;

2. determine whether there is publication bias in favor of significant results;

3. determine the prevalence of practices that support good research practice (study registration, replication studies, data availability); and 
4. provide recommendations for the conduct and reporting of research to minimize publication bias.

\section{METHOD}

To determine the evidential value and level of publication bias, we investigated metaanalyses included in Chow's (2018) article 'Prevalence of Publication Bias Tests in Speech, Language, and Hearing Research'. A pre-registration for this review can be found at: https://osf.io/g3u2p/?view only=ea29e7437f39472e8df5f0a0d59586a2

\section{Selection criteria}

We targeted approximately 50 articles as realistic and achievable based on the time available for the study. From the 29 meta-analyses described in Chow (2018), we focused on four meta-analyses on topics of interest: specific language impairment, developmental speech and language delay/disorder, stuttering, and language interventions. Based on these identified areas, we examined articles from the following four meta-analyses: 'Differences in the Nonword Repetition Performance of Children With and Without Specific Language Impairment: A Meta-Analysis' (Estes et al., 2007), 'The Efficacy of Treatment for Children With Developmental Speech and Language Delay/Disorder: A Meta-Analysis' (Law et al., 2004), 'Behavioural Stuttering Interventions for Children and Adolescents: A Systematic Review and Meta-Analysis' (Nye et al., 2013), and 'The Effectiveness of Parent-Implemented Language Interventions: A Meta-Analysis' (Roberts \& Kaiser, 2011).

Fifty-one articles taken from these 4 meta-analyses were included in this study. Fortythree articles were found via library subscriptions and internet resources; 8 articles were supplied by authors on request. We could not obtain access to 4 articles from these meta-analyses. 


\section{Data Extraction}

Following Simonsohn et al. (Simonsohn et al., 2014), we extracted the inferential statistics corresponding to each article's stated focal (primary) hypothesis. This was more complex than anticipated as many articles had multiple dependent variables, less clearly specified hypotheses, and more complicated designs (e.g. observational or with more than 3 groups). Having identified the focal hypothesis, we identified the corresponding statistical result and recomputed the precise $p$-values based on the reported test statistics. This is necessary where, for example, the value is reported as 'p $<0.05^{\prime}$ instead of reporting ' $\mathrm{p}=0.0369$ ', or where confidence intervals rather than $p$ values were presented. Additionally, $p$-values are sometimes recorded incorrectly (Bakker \& Wicherts, 2011). The full extraction table is included in the OSF repository (details below). Twelve articles were randomly selected for double-extraction, and a further 16 were also double-extracted where the primary coder was uncertain.

Additionally, we searched the articles for evidence of good research practices: study pre-registration, whether the study was a replication of a prior study, and whether data were available.

\section{Data Analysis}

Analyses were completed in R 4.0.5/RStudio 1.4.1106 (R Core Team, 2021; RStudio Team, 2021), using the zcurve package (Bartoš \& Schimmack, 2020a) and modified code for the $p$-curve (Simonsohn, 2017). All scripts and files required to reproduce the analyses for this project are available at the OSF repository: https://osf.io/g53eh/?view only=4504107289e142d3bc438f774b302fb3.

\section{RESULTS}


When we recomputed the $p$-values, we found seven with a more than .01 discrepancy from the original reported values. In one instance, the recomputed $p$-value changed the finding from significant $(\mathrm{p}<.05)$ to non-significant $(\mathrm{p}=.09)$.

\section{$P$-curve results}

Individual $p$-curve analysis was performed on each of the 4 meta-analyses, along with all studies combined (full output in the OSF repository). For the full sample, 112 statistical results were considered, of which 87 were statistically significant. 72 were $p<$ 0.025 . Both the half $p$-curve, $\mathrm{Z}=-17.8, \mathrm{p}<.0001$, and the full $p$-curve were significant, $\mathrm{Z}=$ $-18.3, p<.0001$. As can be seen from Figure 2 , there is a very strong right skew (ie, many more smaller $p$-values than closer to $p=0.05)$. Estimated power from the $p$-curve analysis was $94 \%$. Taken together, these findings suggest that overall there is evidential value in the articles on which meta-analyses reported in ASHA journals are based.

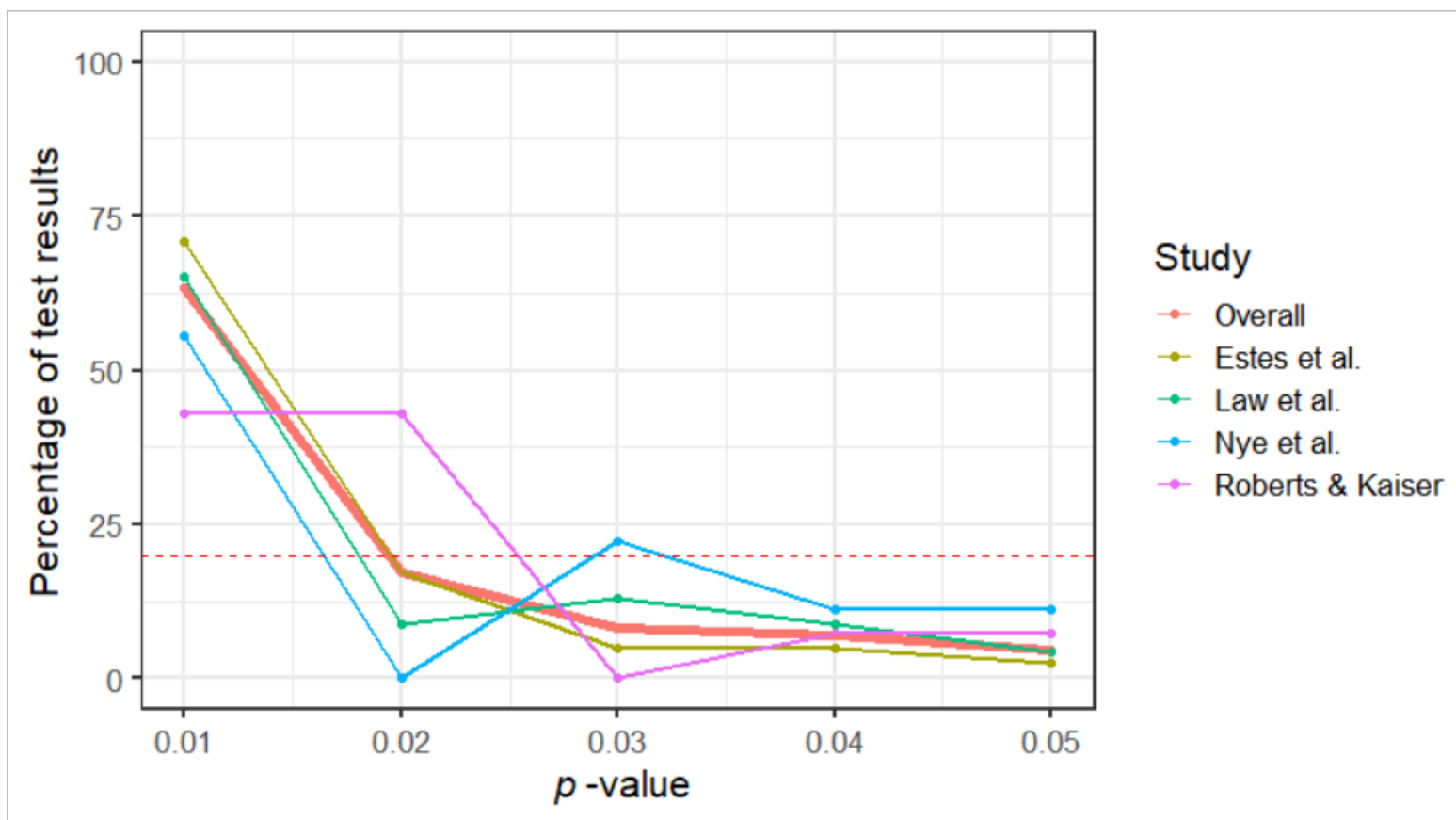

Figure 2: P-curve separately for each meta-analyses, and overall.

Looking at the individual meta-analyses (Figure 2), both Estes et al. and Law et al. had significant right skew for both the half and full $p$-curve, and a clear right skew, with 
estimated power of $98 \%$ and $94 \%$ respectively. These findings all support strong evidential value for the findings of these meta-analyses. In contrast, the evidence for right skew was weaker for Nye et al. and Roberts and Kaiser, with lower estimates for power $(40 \%$ and $60 \%$ respectively).

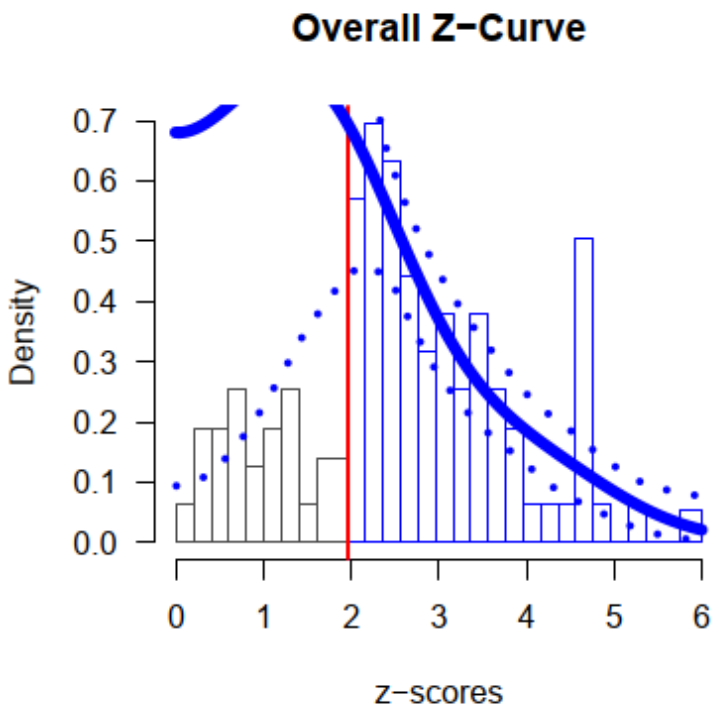

Law et al.

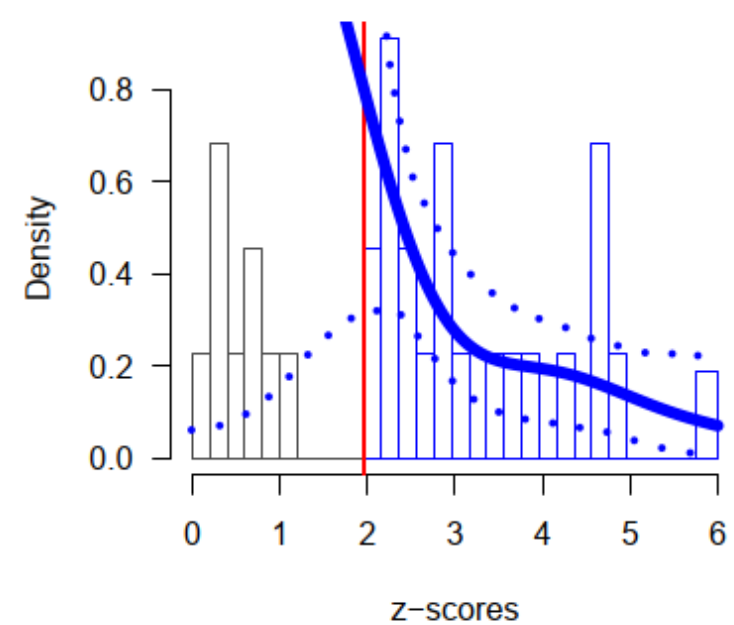

Estes et al.

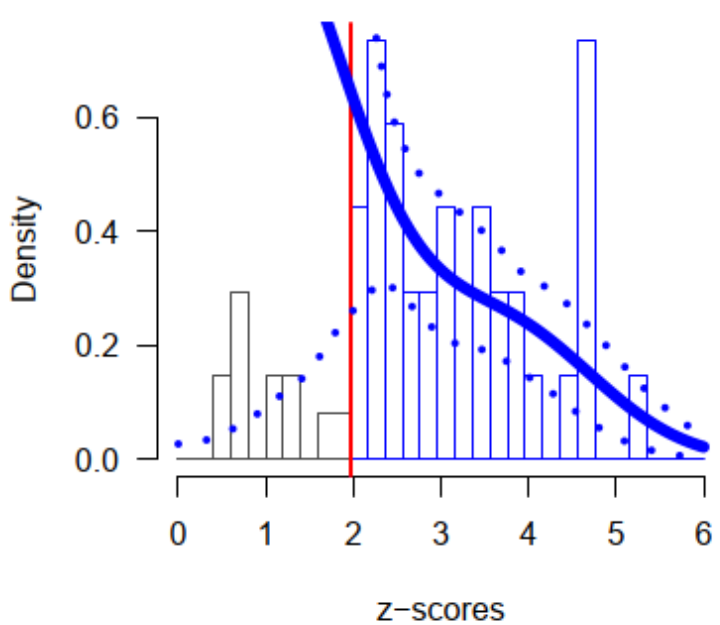

Roberts \& Kaiser

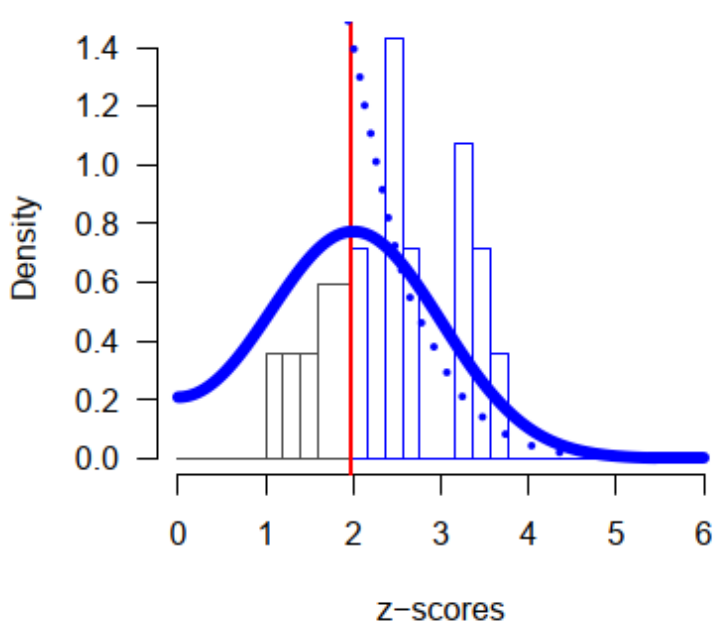

Figure 3: Z-scores separately for each meta-analysis, and overall. Note, there were insufficient scores for Nye et al., so there is no corresponding Z-curve plot. 


\section{Z-curve results}

Results from the $Z$-curve were more pessimistic, with estimated power across all the studies at $43 \%$. As is clear from the top-left panel of Figure 3, there were fewer notquite-significant results reported than would be expected. However, there were a good number of studies reporting very small $p$-values (high $Z$-scores). In contrast to the $p$ curve, studies included in Roberts and Kaiser showed the least evidence of publication bias. The reported results formed a distribution approximating a bell-curve, with more results reported just to the left of the significance criterion. Both Law et al. and Nye et al. had many well powered studies, but there appears to be clearer publication bias with more non-significant results being left unpublished, as evidenced by the lack of papers to the left of $Z=1.96$.

\section{Evidence of Good Research Practices}

As detailed in the supplementary materials on the OSF, none of the 51 studies preregistered their research, none were full replication studies, and none reported either open data or had a data access method. Three studies described themselves as partial replications.

\section{DISCUSSION}

In the 51 papers taken from the four meta-analyses we reviewed, we found no evidence that selective reporting was the sole reason for the significant results found in these studies. This indicates that speech, language and hearing research has evidential value and provides a sound basis for evidence-based practice.

However, results from the $Z$-curve analysis in particular suggest that null findings are less likely to be published in the SLH field. This is consistent with findings from other fields (Fanelli, 2012; Ioannidis et al., 2014; Simonsohn et al., 2014). An alternative 
Pre-print $10^{\text {th }}$ September 2021

interpretation is that many of the included studies had multiple hypotheses and multiple dependent variables. By changing the emphasis between hypotheses, or emphasizing one dependent variable over another, a paper which might have primarily reported a 'null' finding might then report a statistically significant result. With the effort involved in collecting even small samples in specialist populations, it is understandable that researchers want to have their work published. Compared to other documented behaviors, such continuing to collect data until results are significant and outlier exclusion (John et al., 2012), these behaviors are more well-intentioned, but over time may slowly distort the published literature.

For some of the data here, large effects were found, that may limit publication bias. For example, differences in non-word repetition between children with developmental language disorder versus age-matched controls, as documented by the Estes et al. (2007) meta-analysis should find a large effect size. Non-word repetition is now an established clinical marker (IASLT, 2017). The large effect size is reflected in the many highly significant results in both the $p$ - and $Z$-curves. In contrast, some of the intervention studies documented in Nye et al. (2013) and Roberts and Kaiser (2011) may have much smaller effect sizes, and be particularly hard to recruit large samples for. Ensuring that trials with null results are publishable in the SLH literature is imperative in order to get accurate estimates in meta-analyses, and for an unbiased evidence base.

Little evidence was found of good research practices. Preregistration is often seen as a solution to both publication bias by documenting all studies designed, and also limited analytic flexibility — changing the importance of hypotheses or dependent variables. None of the studies here were preregistered. However, $96 \%$ of the studies contained in the meta-analyses were published prior to 2010 , and thus reflect the norms of that time. 
Pre-print $10^{\text {th }}$ September 2021

Since launching in 2012, the Open Science Framework - the largest database to host pre-registrations — has seen preregistrations doubling each year (Kupferschmidt, 2018). Now that pre-registration is simple to do and easy for others to access, this is something SLH researchers should be encouraged to engage in.

Another new marker of open and transparent research practice is sharing data and materials. The OECD have since 2006 had guidelines encouraging open access to publicly funded research data (OECD, 2021), and the European Union has new requirements for open access to publicly funded research data (EU Directive 2019/1024, 2019). Much data from SLH research will require careful consideration with respect to participant anonymity, but there are guidelines for sharing fully anonymized data, or for controlled access to data via data sharing agreements (see e.g., Tucker et al., 2016).

While several of the studies referred to the importance of study replication, no studies were full replication studies, and only 3 studies were partial replications. The Open Science Collaboration (2015) replicated 100 studies published in psychology journals and found only $36 \%$ of studies to produce significant results when the original studies found $97 \%$ significant results. This highlights how important replication studies are to either confirm the original results or to indicate the need for further investigation an this area. This also underlines the importance of publishing null results, including for replications, as this may change evidence-based recommendations.

Limitations of our study include us only including papers from previously published meta-analyses, which means that most of the papers we have included were published prior to 2010. It is also a relatively modest sample size, but the more time-consuming hand-coding of results produces more valid estimates than automated extraction (Simonsohn et al., 2014). The studies we reviewed were also challenging to code, with 
Pre-print $10^{\text {th }}$ September 2021

many having multiple dependent variables and multiple research questions. As noted earlier, SLH studies are often conducted with hard to reach populations, for example children with language impairments, so it is logical to ask multiple research questions when there is access to these populations. A strength of our work was using both the $p$ curve and Z-curve, not relying on a single measure to assess publication bias. Future work in this area could compare the findings from this work with more recently published articles, and a broader cross-section of SLH research.

\section{Recommendations}

We recommend that researchers in SLH should (1) preregister their work, including detailed analytic plans; (2) plan for sharing their data and materials as openly as possible; (3) engage in replication studies; and (4) commit to publishing their studies regardless of whether the primary outcome is statistically significant - at the very least adding it to the grey literature (e.g., as a preprint). Further, journals should work with editors and reviewers to ensure that null results and replication studies are published and valued.

\section{Conclusion}

Research in speech and language therapy has good evidential value — statistically significant effects in published papers are unlikely to be due to chance alone. However, there is also evidence that non-significant findings are less likely to be published, and that the average power of studies is low. Given the challenges of increasing sample sizes in many populations, selecting more powerful designs, running multi-site studies, preregistration and transparent reporting practices will improve the evidence base. Promoting the publication of null findings, in order to minimize publication bias is also important, so that meta-analytic estimates are accurate. 
Pre-print $10^{\text {th }}$ September 2021

\section{ACKNOWLEDGEMENTS}

We thank several authors for responding to requests for unpublished or difficult to find work. 


\section{REFERENCES}

American Speech-Language-Hearing Association. (2005). Evidence-Based Practice in Communication Disorders [Position Statement]. https://www.asha.org/policy/

Bakker, M., \& Wicherts, J. M. (2011). The (mis)reporting of statistical results in psychology journals. Behavior Research Methods, 43(3), 666-678. https://doi.org/10/fdz26x

Bartoš, F., \& Schimmack, U. (2020a). zcurve: An r package for fitting z-curves. https://CRAN.R-project.org/package=zcurve

Bartoš, F., \& Schimmack, U. (2020b). Z-Curve.2.0: Estimating Replication Rates and Discovery Rates. PsyArXiv. https://doi.org/10.31234/osf.io/urgtn

Begg, C. B., \& Mazumdar, M. (1994). Operating characteristics of a rank correlation test for publication bias. Biometrics, 50(4), 1088-1101.

Bishop, D. V. M. (2019). Rein in the four horsemen of irreproducibility. Nature, 568(7753), 435-435. https://doi.org/10/gfztcz

Bishop, D. V. M., \& Thompson, P. A. (2016). Problems in using p-curve analysis and textmining to detect rate of p-hacking and evidential value. PeerJ, 4, e1715. https://doi.org/10/gmbrt7

Brunner, J., \& Schimmack, U. (2020). Estimating Population Mean Power Under Conditions of Heterogeneity and Selection for Significance. Meta-Psychology, 4. https://doi.org/10/gmbrvv

Chow, J. C. (2018). Prevalence of Publication Bias Tests in Speech, Language, and Hearing Research. Journal of Speech, Language, and Hearing Research, 61(12), 3055-3063. https://doi.org/10/gfsf4n

De Rond, M., \& Miller, A. N. (2005). Publish or Perish: Bane or Boon of Academic Life? Journal of Management Inquiry, 14(4), 321-329. https://doi.org/10/bf5k2r 
Pre-print $10^{\text {th }}$ September 2021

Duval, S., \& Tweedie, R. (2000). Trim and Fill: A Simple Funnel-Plot-Based Method of Testing and Adjusting for Publication Bias in Meta-Analysis. Biometrics, 56(2), 455-463. https://doi.org/10/cz42tj

Dwan, K., Gamble, C., Williamson, P. R., \& Kirkham, J. J. (2013). Systematic Review of the Empirical Evidence of Study Publication Bias and Outcome Reporting Bias-An Updated Review. PLOS ONE, 8(7), e66844. https://doi.org/10/f496f7

Egger, M., Smith, G. D., Schneider, M., \& Minder, C. (1997). Bias in meta-analysis detected by a simple, graphical test. BMJ, 315(7109), 629-634. https://doi.org/10/d36qsq

Estes, K. G., Evans, ;l, ,mkj;iioyiuljkjbhhkjn m, \& Else-Quest, N. M. (2007). Differences in the Nonword Repetition Performance of Children With and Without Specific Language Impairment: A Meta-Analysis. Journal of Speech, Language, and Hearing Research, 50(1), 177-195. https://doi.org/10/ftdz27

EU Directive 2019/1024. (2019, June 20). PE/28/2019/REV/1, Directive (EU) 2019/1024 of the European Parliament and of the Council of 20 June 2019 on open data and the re-use of public sector information [Website]. Publications Office of the European Union. http://op.europa.eu/en/publication-detail//publication/a6ef4c41-97eb-11e9-9369-01aa75ed71a1

Fanelli, D. (2012). Negative results are disappearing from most disciplines and countries. Scientometrics, 90(3), 891-904. https://doi.org/10/bq24dv

Greenland, S. (1994). Invited Commentary: A Critical Look at Some Popular MetaAnalytic Methods. American Journal of Epidemiology, 140(3), 290-296. https://doi.org/10/gjqbfx

Higgins, J. P. T., Savović, J., Page, M. J., Elbers, R. G., \& Sterne, J. A. C. (2021). Assessing risk of bias in a randomized trial. In J. P. T. Higgins, J. Thomas, J. Chandler, M. Cumpston, T. Li, M. J. Page, \& V. A. Welch (Eds.), Cochrane Handbookfor Systematic Reviews of Interventions (6.2). www.training.cochrane.org/handbook. 
IASLT. (2017). Supporting Children With Developmental Language Disorder In Ireland (IASLT Position Paper And Guidance Document). https://www.iaslt.ie/documents/public-information/Childhood Speech and Language/DLD Position Paper FINAL 23MAY2017.pdf

Ioannidis, J. P. A. (2005). Why Most Published Research Findings Are False. PLOS Medicine, 2(8), e124. https://doi.org/10/chhf6b

Ioannidis, J. P. A., Munafò, M. R., Fusar-Poli, P., Nosek, B. A., \& David, S. P. (2014). Publication and other reporting biases in cognitive sciences: Detection, prevalence, and prevention. Trends in Cognitive Sciences, 18(5), 235-241. https://doi.org/10/f5345b

John, L. K., Loewenstein, G., \& Prelec, D. (2012). Measuring the Prevalence of Questionable Research Practices With Incentives for Truth Telling. Psychological Science, 23(5), 524-532. https://doi.org/10/f33h6z

Kupferschmidt, K. (2018, September 21). More and more scientists are preregistering their studies. Should you? Science | AAAS. https://www.sciencemag.org/news/2018/09/more-and-more-scientists-arepreregistering-their-studies-should-you

Law, J., Garrett, Z., \& Nye, C. (2004). The Efficacy of Treatment for Children With Developmental Speech and Language Delay/Disorder. Journal of Speech, Language, and Hearing Research, 47(4), 924-943. https://doi.org/10/fq2zxg

Light, R. J., \& Pillemer, D. B. (1984). Summing up: The science of reviewing research. Cambridge, Mass. : Harvard University Press. http://archive.org/details/summingupscience00ligh Macaskill, P., Walter, S. D., \& Irwig, L. (2001). A comparison of methods to detect publication bias in meta-analysis. Statistics in Medicine, 20(4), 641-654. https://doi.org/10/fwtz3c 
Nye, C., Vanryckeghem, M., Schwartz, J. B., Herder, C., Turner, H. M., \& Howard, C. (2013). Behavioral Stuttering Interventions for Children and Adolescents: A Systematic Review and Meta-Analysis. Journal of Speech, Language, and Hearing Research, 56(3), 921-932. https://doi.org/10/gmbg6z

OECD. (2021). Recommendation of the OECD Council concerning Access to Research Data from Public Funding. https://www.oecd.org/sti/recommendation-access-toresearch-data-from-public-funding.htm

Open Science Collaboration. (2015). Estimating the reproducibility of psychological science. Science, 349(6251). https://doi.org/10/68c

R Core Team. (2021). R: A language and environment for statistical computing [Manual]. https://www.R-project.org/

Roberts, M. Y., \& Kaiser, A. P. (2011). The Effectiveness of Parent-Implemented Language Interventions: A Meta-Analysis. American Journal of Speech-Language Pathology, 20(3), 180-199. https://doi.org/10/fr9j4k

Rosenthal, R. (1979). The file drawer problem and tolerance for null results. Psychological Bulletin, 86(3), 638-641. https://doi.org/10/d5sxt3

RStudio Team. (2021). RStudio: Integrated development environment for $r$ [Manual]. http://www.rstudio.com/

Simmons, J. P., Nelson, L. D., \& Simonsohn, U. (2011). False-Positive Psychology: Undisclosed Flexibility in Data Collection and Analysis Allows Presenting Anything as Significant. Psychological Science, 22(11), 1359-1366. https://doi.org/10/bxbw3c

Simonsohn, U. (2017). P-curve 4.06. http://p-curve.com/app4/pcurve_app4.06.r Simonsohn, U., Nelson, L. D., \& Simmons, J. P. (2014). P-curve: A key to the file-drawer. Journal of Experimental Psychology: General, 143(2), 534-547. https://doi.org/10/gffnn9 
Pre-print $10^{\text {th }}$ September 2021

Sterling, T. D. (1959). Publication Decisions and their Possible Effects on Inferences Drawn from Tests of Significance-Or Vice Versa. Journal of the American Statistical Association, 54(285), 30-34. https://doi.org/10/gckf9z

Tucker, K., Branson, J., Dilleen, M., Hollis, S., Loughlin, P., Nixon, M. J., \& Williams, Z. (2016). Protecting patient privacy when sharing patient-level data from clinical trials. BMC Medical Research Methodology, 16(1), 77. https://doi.org/10/gmcf94 